\section{Exporting Death Made in Quebec}

The decision by the Quebec government to continue exporting chrysotile asbestos is a public health tragedy for Canada and the rest of the world. Asbestos kills workers and citizens. It has proven impossible to devise practical methods of safely manipulating asbestos. This has led over 40 countries, including the European Union, to ban chrysotile. However, it continues to be exported, mostly to Low and Middle Income Countries (LMIC), particularly in Asia, by countries at the forefront of public health such as Zimbabwe, Russia, Kazakhstan and Canada.

Thousands of workers will die as a result of exposure to Quebec's chrysotile asbestos which is used mostly in cement for the construction industry. In addition to having no protective equipment, these workers will have little if any medical, legal or social protection when they develop the asbestos-related diseases since the health care systems of these LMIC are poorly equipped to diagnose and treat patients with these conditions and workers compensation systems are often non-existent or ineffectual. As buildings age and the cement starts to erode and crumble, citizens will become exposed to sufficient concentrations of fibres to produce deleterious health effects. The death toll will rise substantially above the current estimate of 100,000 annual deaths among exposed workers for decades to come, given the size of the populations potentially exposed in Asia and the latency period of 40 or more years for many asbestos-related diseases.

While Canada and Quebec are removing all forms of asbestos from public buildings and while its use is all but halted in Quebec as in most of the developed world, the government invests hundreds of thousands of dollars in the Chrysotile Institute, a front for the industry, which disseminates biased information on product safety to the media and foreign governments. In addition, Canada has gladly done Quebec's bidding and successfully prevented chrysotile from being included in the Rotterdam Convention, the United Nations registry of hazardous materials.

The decision is wrong, unethical, indecent and we should be outraged. But we shouldn't be surprised. Quebec's export of asbestos to India alone is worth over 400 million dollars per year. With the mesotheliomas, the other cancers, the asbestosis, the deaths and suffering to workers and their families occurring far away from the public's eye, this is a good deal for government.

What next? Maybe Quebec can nationalize its tobacco industry and start pushing cigarettes to teenage girls in Asia.

Gilles Paradis

Scientific Editor

\section{Exporter la mort fabriquée au Québec}

La décision du gouvernement québécois de continuer à exporter de l'amiante chrysotile est une tragédie sanitaire pour le Canada et le reste du monde. L'amiante tue des travailleurs et des citoyens. Il s'est avéré impossible de trouver des méthodes pratiques de le manipuler sans danger. C'est ce qui a poussé une quarantaine de pays, dont ceux de l'Union européenne, à interdire le chrysotile. Pourtant, des pays aux premières lignes de la santé publique (le Zimbabwe, la Russie, le Kazakhstan, le Canada) continuent à en exporter, principalement vers les pays à faible revenu et à revenu intermédiaire (PFRRI), surtout en Asie.

Des milliers de travailleurs mourront d'avoir été exposés à l'amiante chrysotile du Québec, que l'on utilise surtout dans le ciment destiné à l'industrie du bâtiment. En plus de ne porter aucun équipement de protection, ces travailleurs n'auront presque aucun recours médical, juridique ou social lorsqu'ils contracteront les maladies liées à l'amiante. Dans les PFRRI, les systèmes de santé sont mal équipés pour diagnostiquer et traiter les patients qui présentent ces maladies, et les systèmes d'indemnisation des travailleurs sont souvent inefficaces ou inexistants. Quand les bâtiments vieilliront et que leur ciment commencera à s'éroder et à se désagréger, les citoyens seront exposés à des concentrations de fibres d'amiante suffisantes pour avoir des effets néfastes sur leur santé. Dans les décennies à venir, le bilan des pertes humaines, qui est actuellement de 100000 décès par année, devrait considérablement s'alourdir chez les travailleurs exposés, étant donné la taille des populations potentiellement vulnérables en Asie et la période de latence de 40 ans ou plus pour de nombreuses maladies liées à l'amiante.

Pendant que le Canada et le Québec retirent toutes les formes d'amiante des édifices publics et que l'utilisation du produit s'est pratiquement arrêtée au Québec comme dans la plupart des pays développés, le gouvernement investit des centaines de milliers de dollars dans l'Institut du chrysotile, un prête-nom pour l'industrie, qui diffuse des informations biaisées sur la sécurité du produit aux médias et aux gouvernements étrangers. Mieux encore : le Canada a été heureux d'accéder à la demande du Québec en empêchant que le chrysotile figure dans la Convention de Rotterdam, le registre onusien des matières dangereuses.

C'est une mauvaise décision, indécente et contraire à l'éthique. Elle devrait nous scandaliser, mais non nous étonner. Les exportations québécoises d'amiante vers l'Inde représentent à elles seules plus de 400 millions de dollars par année. Puisque les mésothéliomes et autres cancers, l'asbestose, les décès et la souffrance des travailleurs et de leurs familles se produisent très loin des yeux de ses citoyens, c'est une bonne affaire pour le gouvernement.

Après cela, peut-être que le Québec pourrait nationaliser son industrie du tabac et vanter les mérites de la cigarette auprès des adolescentes en Asie...

Le rédacteur scientifique, Gilles Paradis 